\title{
A non-local formulation for level-set modelling
}

\author{
Stéphane Valance* \\ Nuclear Energy and Safety, Paul Scherrer Institut, 5232 Villigen PSI, Switzerland
}

\begin{abstract}
The level-set method, as introduced by Osher et al. (Osher, S., \& Sethian, J., 1988. Fronts propagating with curvature-dependent speed: Algorithms based on Hamilton-Jacobi formulations. Journal of Computational Physics, 79, 12-49.) presents some flaws with respect to algorithmic simplicity and solution admissibility. In this paper, we present a robust approach for the level-set modelling by taking advantage of readily available work from phase-field framework. By adapting the latter with proper velocity correction, we have obtained a non-local formulation of the level-set problem that overrides the aforementioned problems. An application of this approach, both in explicit finite differences and implicit iso-geometric analysis, illustrates the efficiency of this new formulation.
\end{abstract}

La méthode level-set (fonction de niveaux), telle qu'introduite par Osher et al. (Osher, S., \& Sethian, J., 1988. Fronts propagating with curvature-dependent speed: Algorithms based on Hamilton-Jacobi formulations. Journal of Computational Physics, 79, 12-49.) présente des inconvénients lorsque l'on s'intéresse à la simplicité algorithmique et á l'admissibilité des solutions. Dans cet article, nous présentons une approche robuste pour la modélisation par fonctions de niveaux en s'inspirant de développements déjà disponibles réalisé dans le cadre des méthodes de champs de phases. En adaptant cette approche avec une correction appropriée de la vitesse, nous avons obtenu une formulation non-locale du problème levelset qui permet de s'affranchir des difficultés citées plus haut. La méthode développée est appliquée à un cas test standard, à la fois en différences finies avec un schéma explicite et dans le cadre iso-géométrie avec un schéma implicite, illustrant ainsi son efficacité et sa versatilité.

Keywords: level-set; iso-geometry

Mots-clés: fonction de niveaux; iso-géometrie

\section{Modelling hyper-surfaces}

In the level-set approach, hyper-surfaces are modelled through an implicit description. In the framework of structural mechanics, this approach encountered a rising success past decade as they were used for the modelling of cracks (Gravouil, Moës, \& Belytschko, 2002) and phase change interfaces (Coret, Valance, Laniel, \& Réthoré, 2009; Harari \& Dolbow, 2010) in the eXtended Finite Element Method (X-FEM).

However, the level-set technique, as introduced by Osher and Sethian (1988), is not easy to handle: it implies three different equations - i.e. level-set propagation, velocity extension and signed distance reinitialisation - at not a priori predictable moments of the computation.

*Email: Stephane.Valance@psi.ch 
Moreover, the propagation equation turns out to be difficult to solve in the classical Galerkin set-up without proper stabilisation terms (Chessa, Smolinski, \& Belytschko, 2002; Valance, De Borst, Réthoré, \& Coret, 2008).

In this work, we propose a non-local level-set formulation that enables to suppress the aforementioned problems: only one equation is necessary to handle correctly the level-set surface propagation. The velocity field is still to be known on the domain of interest, but there is no need any more to enforce the orthogonality between the field lines of the level-set field and the field lines of the normal velocity field.

The paper is structured as follows. First, we will recall the basics of level modelling. We then introduce the approach used to formulate a non-local evolution equation for level-set modelling. Afterwards, we present the numerical set-up used to study this model in both finite differences and finite elements - iso-geometry - approaches. Finally, we discuss results obtained by the two techniques in the classic benchmark of a rigid motion of a circular iso- 0 .

\section{Definition of a level-set}

The space that defines level-set over a domain $\mathcal{D}$ in the sequel is the space $\mathcal{L S}$ such that:

$$
\mathcal{L S}=\left\{\phi \in \mathcal{C}^{1}(\mathcal{D} \rightarrow \mathbb{R}) \mid \forall \underline{M} \in \mathcal{D}, \phi_{\underline{\underline{M}}}=0 \Rightarrow\|\underline{\nabla} \phi\|_{\underline{\underline{M}}} \neq 0\right\} .
$$

The definition of a surface $S_{\phi}$ on a level-set field $\phi$ of the above space is a smooth surface characterised by:

$$
\forall \phi \in \mathcal{L} \mathcal{S}, S=\left\{\underline{M} \in \mathcal{D} \mid \phi_{\mid \underline{M}}=0\right\}
$$

If the level-set field is now attached to a particle field $\underline{x}$,

$$
\phi=\hat{\phi}(t, \underline{x}(t))
$$

it follows from the stationarity of the level-set field with respect to the particle field $\underline{x}$ :

$$
\frac{\mathrm{D} \phi}{\mathrm{D} t}=0 \Leftrightarrow \frac{\partial}{\partial t} \hat{\phi}(t, \underline{x}(t))+\frac{\partial \underline{x}(t)}{\partial t} \cdot \underline{\nabla} \hat{\phi}(t, \underline{x}(t)) \Leftrightarrow \frac{\partial \phi}{\partial t}+F\|\underline{\nabla} \phi\|=0
$$

where, $\|\bullet\|$ is the euclidean norm of $\bullet F$ is an external data of the problem defined with respect to the required surface motion at velocity $\underline{u}_{\mathcal{S}}$ of the surface $\mathcal{S}_{\phi}$ by:

$$
F \in \mathcal{C}(\mathcal{D} \rightarrow \mathbb{R}), \quad F_{\mid \underline{M} \in S}:=\underline{u}_{\mathcal{S}} \cdot \frac{\underline{\nabla} \phi}{\|\underline{\nabla} \phi\|}=\frac{\partial \underline{x}(t)}{\partial t} \cdot \frac{\underline{\nabla} \phi}{\|\underline{\nabla} \phi\|}
$$

Equation (4) is the standard propagation equation of the surface $\mathcal{S}_{\phi}$ implicitly defined by the level-set field $\phi$.

\section{Order parameter: characteristic thickness and motion}

To establish a non-local form of the above introduced propagation equation, we will make an extensive use of already derived results obtained in the framework of phase change theory (Cahn \& Allen, 1977). The Allen-Cahn equation for the modelling of ordering phenomenon in continuum media via a field $\eta$ reads: 


$$
\frac{\partial \eta}{\partial t}+M\left(\psi^{\prime}(\eta)-\kappa \Delta \eta\right)=0
$$

where, $M$, the mobility coefficient, and $\kappa$, the interfacial energy coefficient, are parameters of the model; $\psi$ is a function associating energy states to values of $\eta$.

The above equation arises as the solution of the minimisation of a potential energy field defined as follows:

$$
E(\eta)=\psi(\eta)+\frac{\kappa}{2} \underline{\nabla} \eta \cdot \underline{\nabla} \eta
$$

In the above definition, the technical definition of $\psi$ is a prototypical double-well potential: it possess two local minima at $\left\{-\eta_{\mathrm{e}} ; \eta_{\mathrm{e}}\right\}$ and a local maximum at 0 valued to $w_{\mathrm{b}}$. The local minima correspond to a stable state. The local maximum represents an energy barrier that is to be crossed in order to change from one phase to another. Finally, the second term in Equation (7) provides an interfacial energy characteristic of the two phases interaction.

A slightly modified form of the Cahn-Allen equation is widely known as the GinzburgLandau equation. Caginalp (1986) analysis demonstrated the existence of solutions for this equation and that the thickness of the interface remains stable throughout time. Our purpose is now to adapt this equation in order to build a level-set propagation equation where the level-field is ensured to remain in $\mathcal{L}$.

In the order-parameter context, an interface as an infinite thickness. However, it is possible to define a relevant measure of the interface thickness $e$ through:

$$
e=2 \frac{\eta_{\mathrm{e}}}{\|\underline{\nabla} \eta\|_{\eta=0}}
$$

With such a definition, at equilibrium, the following relation between the $\kappa$ parameter and the thickness holds:

$$
\kappa=\frac{w_{\mathrm{b}}}{2}\left(\frac{e}{\eta_{\mathrm{e}}}\right)^{2}
$$

For a logarithmic double-well potential, the velocity $F_{\mathrm{K}}$ of the iso- 0 surface in the absence of external action is given by (Cahn \& Allen, 1977):

$$
F_{\mathrm{K}}=M \bar{K}
$$

where, $\bar{K}$ is the mean curvature of the iso- 0 surface of the order parameter field.

If the order parameter field is attached to a particle field $\underline{x}$ moving at the velocity $F-M \bar{K}$, we obtain the propagation equation of a level-set field at the velocity $F$ which verifies the belonging of the field $\phi$ to the space $\mathcal{L S}$. After some reduction, using the formula for curvature proposed in Goldman (2005), one obtains the equation:

$$
\frac{\partial \phi}{\partial t}+F\|\underline{\nabla} \phi\|+M\left(\psi^{\prime}(\phi)-\frac{\kappa}{\underline{\nabla} \phi \cdot \underline{\nabla} \phi} \underline{\nabla} \phi \cdot \underline{\underline{H}} \phi \cdot \underline{\nabla} \phi\right)=0,
$$

where, $\underline{H} \bullet$ represents the Hessian differential operator applied to $\bullet$.

Equation (11) is a non-local propagation equation for a level-set field $\phi$. It can already be seen that this equation includes one more term with respect to the usual propagation equation, 


$$
M\left(\psi^{\prime}(\phi)-\frac{\kappa}{\underline{\nabla} \phi \cdot \underline{\nabla} \phi} \underline{\nabla} \phi \cdot \underline{\underline{H}} \phi \cdot \underline{\nabla} \phi\right) .
$$

Considering a zero normal velocity, the equilibrium, i.e. $\partial \phi / \partial t=0$, is reached when this term collapses. This term can, therefore, be considered as a measure of the quality of the gradient of the level-set field at the interface. In the sequel, we will use this term in order to control the spatial quality of the obtained solution. Therefore, we introduce the following definitions:

$$
\begin{gathered}
f^{\mathrm{p}}(\phi):=\|\underline{\nabla} \phi\|, \\
f^{\mathrm{g}}(\phi):=\left(\psi^{\prime}(\phi)-\frac{\kappa}{\underline{\nabla} \phi \cdot \underline{\nabla} \phi} \underline{\nabla} \phi \cdot \underline{\underline{H}} \phi \cdot \underline{\nabla} \phi\right) .
\end{gathered}
$$

\section{Numerical implementation}

\subsection{Explicit finite differences}

The scheme presented below is an explicit finite difference scheme. The equation is solved on a two-dimensional domain regularly gridded with a grid step size $h$. Two orders of truncation have been tested to compute the spatial differential operator. The first one, denoted as low-order scheme, uses a 5 points stencil and presents a truncation error of $\mathcal{O}\left(h^{2}\right)$ for both first and second derivatives. The second one, high-order scheme, uses a 16 points stencil and presents a truncation error of $\mathcal{O}\left(h^{4}\right)$ for both first and second derivatives.

The time integration scheme is the standard embedded Runge-Kutta at fourth order. We use here, the Cash-Karp (Cash \& Karp, 1990) version that enables to estimate the time integration error. Let $\hat{\phi}_{n}$ be the nodal vector associated to the level-set field at time step $n$. The explicit time integration scheme is:

$$
\underset{\sim}{\hat{\phi}_{n+1}}=\underset{\sim}{\hat{\phi}_{n}}+\sum_{i=1}^{6} c_{i} \hat{\sim}_{i}
$$

where, the $\underset{\sim}{\hat{k}_{i}}$ at node $A,\left(\underset{\sim}{\hat{k}_{i}}\right)_{A}$, is defined by:

$$
\left(\begin{array}{c}
\hat{k}_{i} \\
\sim
\end{array}\right)_{A}=\Delta t\left(F \cdot f^{\mathrm{p}}\left(\left(\underset{\sim}{\hat{\phi}_{n}}+\sum_{j=1}^{i-1} b_{i j} \underset{\sim}{\hat{k}_{j}}\right)_{A}\right)+M \cdot f^{\mathrm{g}}\left(\left(\underset{\sim}{\hat{\phi}_{n}}+\sum_{j=1}^{i-1} b_{i j} \hat{\sim}_{j}\right)\right)\right),
$$

with $\Delta t$ the current time step length and $\phi_{A}$ being the value of field $\hat{\phi}$ at node $A$. The parameters can be found in Cash and Karp (1990).

With this approach, an error estimate $\Delta_{n}$ for time integration at time step $n$ can be obtained through:

$$
\Delta_{n}=\left|\sum_{i=1}^{6}\left(c_{i}-c_{i}^{\star}\right) \cdot \underset{\sim}{\hat{k}_{i}}\right|
$$

The structure of the non-local propagation equation authorises to define an error estimate of the quality of the solution with respect to the interface gradient, $\Theta_{n}$, through: 


$$
\Theta_{n}=\left|\hat{f}_{\sim}^{g}(\underset{\sim}{\hat{\phi}})\right|,
$$

with, $\hat{f}^{g}$ being defined at node $A$ by:

$$
\left(\hat{f}_{\sim}^{g}\left(\begin{array}{c}
\hat{\phi} \\
\sim
\end{array}\right)\right)_{A}=f^{\mathrm{g}}\left((\underset{\sim}{\hat{\phi}})_{A}\right) .
$$

\subsection{Implicit Galerkin}

The variational problem associated to the above introduced non-local propagation equation is as follows:

$$
\begin{gathered}
\left(\mathcal{P}_{20}\right) \forall q \in \mathcal{U}_{\varpi}^{0} \text {, find } \phi \in \mathcal{U}_{\varpi} \text { such that: } \\
\qquad \mathcal{B}_{\phi}(q, \phi)=0
\end{gathered}
$$

where,

$$
\begin{aligned}
\mathcal{B}_{\phi}(q, \phi) & :=\mathcal{B}^{t}(q, \phi)+\mathcal{B}^{\mathrm{p}}(q, \phi)+\mathcal{B}^{\mathrm{g}}(q, \phi) \\
& =\left\langle q, \phi_{, t}\right\rangle_{\Omega}+\left\langle q, F f^{\mathrm{p}}(t, \phi)\right\rangle_{\Omega}+\left\langle q, M f^{\mathrm{g}}(t, \phi)\right\rangle_{\Omega}
\end{aligned}
$$

and the spaces $\mathcal{U}_{\varpi}^{0}$ and $\mathcal{U}_{\varpi}$ are defined by:

$$
\begin{aligned}
& \mathcal{U}_{\varpi}=\left\{\varrho \in H^{1}(\Omega) \mid \forall \underline{M} \in \Gamma_{\varpi}, \varrho=\varpi\right\}, \\
& \mathcal{U}_{\varpi}^{0}=\left\{\varrho \in H^{1}(\Omega) \mid \forall \underline{M} \in \Gamma_{\varpi}, \varrho=0\right\} .
\end{aligned}
$$

The time discretisation of the problem is done using the generalised- $\alpha$ method developed by Chung and Hulbert (1993). This method has already been used in the framework of isogeometric modelling to solve phase-field type problems (Gómez, Calo, Bazilevs, \& Hughes, 2008). The discretised vector $\Upsilon$ is defined by:

$$
N_{\sim}^{\mathrm{T}} \cdot \underset{\sim}{\Phi} \simeq \phi
$$

where, $N^{\mathrm{T}}$ is the vector of shape functions expressed at the nodes. In the generalised- $\alpha$ approach, time derivatives are estimated at different time within the actual time step; we refer to these time derivatives, including their derivation time, using $\left(\underset{\sim}{\ddot{\Phi}_{n+\alpha_{\mathrm{m}}}}, \underset{\sim}{\dot{\Phi}_{n+\alpha_{\mathrm{f}}}}, \underset{\sim}{\Phi_{n+\alpha_{\mathrm{f}}}}\right)$ where, $\alpha_{\mathrm{f}}$ and $\alpha_{\mathrm{m}}$ are parameters. The definition of the residual vector is then:

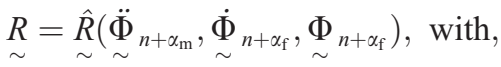

$$
\begin{aligned}
& R=\left\{\mathcal{B}\left(N_{\mathrm{A}}, \phi^{\mathrm{h}}\right)\right\} .
\end{aligned}
$$


The time-space discreticed problem then reads:

$$
\left(\mathcal{P}_{25}\right) \text { given } \ddot{\sim}_{n}, \dot{\sim}_{n}, \underset{\sim}{\Phi},
$$

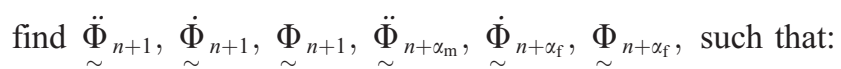

$$
\underset{\sim}{\hat{R}}\left(\ddot{\sim}_{n+\alpha_{\mathrm{m}}}, \dot{\sim}_{n+\alpha_{\mathrm{m}}}, \underset{\sim}{\Phi_{n+\alpha_{\mathrm{f}}}}\right)=\underset{\sim}{0}
$$

where,

$$
\begin{aligned}
& \underset{\sim}{\Phi}{ }_{n+1}={\underset{\sim}{\Phi}}_{n}+\Delta t_{n}{\underset{\sim}{\Phi}}_{n}+\frac{\Delta t_{n}^{2}}{2} \ddot{\sim}_{n}+\beta \Delta t_{n}^{2}\left(\ddot{\sim}_{n+1}-{\underset{\sim}{\Phi}}_{n}\right), \\
& {\underset{\sim}{\Phi}}_{n+1}=\dot{\sim}_{n}+\Delta t_{n} \ddot{\sim}_{n}+\gamma \Delta t_{n}\left(\ddot{\sim}_{\sim} \ddot{\sim}_{n+1}-\ddot{\sim}_{n}\right), \\
& {\underset{\sim}{\Phi}}_{n+\alpha_{\mathrm{m}}}={\underset{\sim}{\Phi}}_{n}+\alpha_{\mathrm{m}}\left({\underset{\sim}{\Phi}}_{n+1}-\ddot{\sim}_{n}\right), \\
& {\underset{\sim}{\Phi}}_{n+\alpha_{\mathrm{f}}}=\dot{\sim}_{n}+\alpha_{\mathrm{f}}\left({\underset{\sim}{\Phi}}_{n+1}-\dot{\sim}_{n}\right), \\
& \underset{\sim}{\Phi_{n+\alpha_{\mathrm{f}}}}=\underset{\sim}{\Phi_{n}}+\alpha_{\mathrm{f}}\left({\underset{\sim}{\Phi_{n+1}}}-{\underset{\sim}{\Phi}}_{n}\right) .
\end{aligned}
$$

The non-linear discretised problem $\left(\mathcal{P}_{25}\right)$ is solved using a predictor-corrector algorithm. In this algorithm, the prediction step is done under the "same $\Phi$ " hypothesis (Jansen, Whiting, \& Hulbert, 2000).

Here, a control of the time integration precision is also implemented: two successive computations of the same time step are conducted. The first one is done with every generalised- $\alpha$ parameters equal to 1, which renders the scheme equivalent to a backward Euler scheme. The second one is conducted with $\rho_{\infty}=\frac{1}{2}$ and other parameters chosen following Bazilevs, Calo, Zhang, and Hughes (2006). The two schemes present a difference of one order in integration precision. An estimation of the time integration precision $\Delta_{n}$ at time step $n$ is obtained by comparing the two obtained residual vectors:

$$
\Delta_{n}=\left|\underset{\sim}{\Phi_{n}^{\mathrm{BE}}}-\underset{\sim}{\Phi_{n}^{\alpha}}\right|,
$$

where, $\Phi_{n}^{\mathrm{BE}}$ and $\Phi_{n}^{\alpha}$ are the solutions obtained using the backward Euler and the generalised$\alpha$ schemes, respectively.

As in the finite differences case, it is possible to introduce an estimate of the quality of the gradient norm through $\Theta_{n}$ defined by:

$$
\Theta_{n}=\left|\left\{\mathcal{B}^{\mathrm{g}}\left(N_{\mathrm{A}}, \phi^{\mathrm{h}}\right)\right\}\right|
$$




\subsection{Time and space quality control}

In the derived equation for the non-local propagation of the level-set field, 11, the second term on the right hand side enables to get an estimate of the quality of the gradient norm at the interface. Otherwise said, the norm of this term is directly proportional to the error with respect to the stationary solution of the problem for the actual location of the iso- 0 surface.

A look at this term shows that the modification of the parameter $M$ will enable to enforce the control of this error throughout the computation via a control approach of the form:

$$
M_{n+1}=M_{n}\left(\mathcal{S}^{\mathrm{g}}\left(\frac{\Theta_{0}}{\Theta_{n}}\right)^{\beta^{\mathrm{g}}}\right)^{-1}
$$

where, $\mathcal{S}^{\mathrm{g}}$ is a safety coefficient slightly smaller than the unit, $\Theta_{0}$ is the target error on the gradient to be estimated at the beginning of the computation and $\beta^{\mathrm{g}}$ is a parameter that may vary depending if the target error is exceeded or not.

This control of the parameter $M$ enables to set the velocity at which the control of the gradient is done relatively to the overall propagation velocity of the field, $F$.

The control of the time step length can be achieved in both explicit and implicit schemes through the use of the time integration error estimate. Several functions have been determined for such a control (Gustafsson, 1991, 1994; Söderlind, 2006). In our approach, we selected a simple, but reliable version:

$$
\Delta_{n+1}=\mathcal{S}^{\mathrm{t}}\left(\frac{\Delta_{0}}{\Delta_{n}}\right)^{\beta^{t}} .
$$

where, $\mathcal{S}^{t}$ is a safety coefficient slightly smaller than the unit, $\Delta_{0}$ is the target error in gradient to be estimated at the beginning of the computation and $\beta^{t}$ is a parameter that may vary depending if the target error has been exceeded or not.

\section{Benchmark}

\subsection{Rigid motion test set-up}

One of the difficult motions to simulate within level-set is the rigid body motion: a hypersurface is submitted to a velocity field that does not involve shape change. Classical test case in this area is the simulation of the rotation of a circle shape for half a revolution. In the sequel, our computational domain is a square plate of unit size with its centre being located in $(0 ., 0$. $)$ in Cartesian coordinates. In the rigid body motion test case, we initialise the levelset field through the projection of the field defined by:

$$
(x+.25)^{2}+y^{2}=(.125)^{2},
$$

where, $(x, y)$ are the Cartesian coordinates of the current point. The velocity is then defined by:

$$
F=\underline{M} \cdot \underline{\nabla} \phi,
$$

where, $\underline{M}$ is the coordinate of the current point taken from the centre of the plate. 
In these tests, the error on the solution is measured by comparison to a numerical ideal solution. This reference solution is obtained by initialising the computation with a circular contour centred at $(.25,0$. $)$ and then obtaining the complete field by using the propagation equation with a null velocity. This error is hereafter denoted relative error.

The double-well potential $\psi$ is constructed using the logarithmic potential:

$$
\psi(x)=x \log (x)+(1-x) \log (1-x)+3 x(1-x)
$$

usually used in the phase-field theory. This usual double-well potential is scaled such that the two local minimums are located at $\{-1 ; 1\}$ and are valued to 0 . For numerical reasons, it is extended by continuity at fourth order with a polynomia outside the minimums locations, i.e. on $]-\infty ;-1[$ and on $] 1 ; \infty[$. Due to its non-significance in the current context, the local maximum at 0 is valued to 1 and, therefore, the parameter $w_{\mathrm{b}}$ is also valued to 1 .

\subsection{Finite differences}

In the case of finite differences, we have tested two different ways of selecting the parameter $\kappa$ : either using a constant value - corresponding to an interface thickness of .1 via Equation (8) - or a value evolving with the grid spacing - corresponding to an interface thickness of four times the grid spacing.

In the case of a thickness of the interface varying with the mesh refinement, it can be seen in Figure 1 that there is a lower limit for the relative error. This limit characterises the inability to correctly compute the derived quantities when the interface becomes too thin. As a result, even with finer meshes, the solution contour presents a constant shift with respect to the theoretic solution contour as displayed in Figure 2. However, the shape of the contour, for high order and/or fine grid is close to the ideal shape.

Figure 3 displays the relative error for a constant interface thickness and low-order and high-order schemes. The final obtained contour compared to the theoretic solution contour is

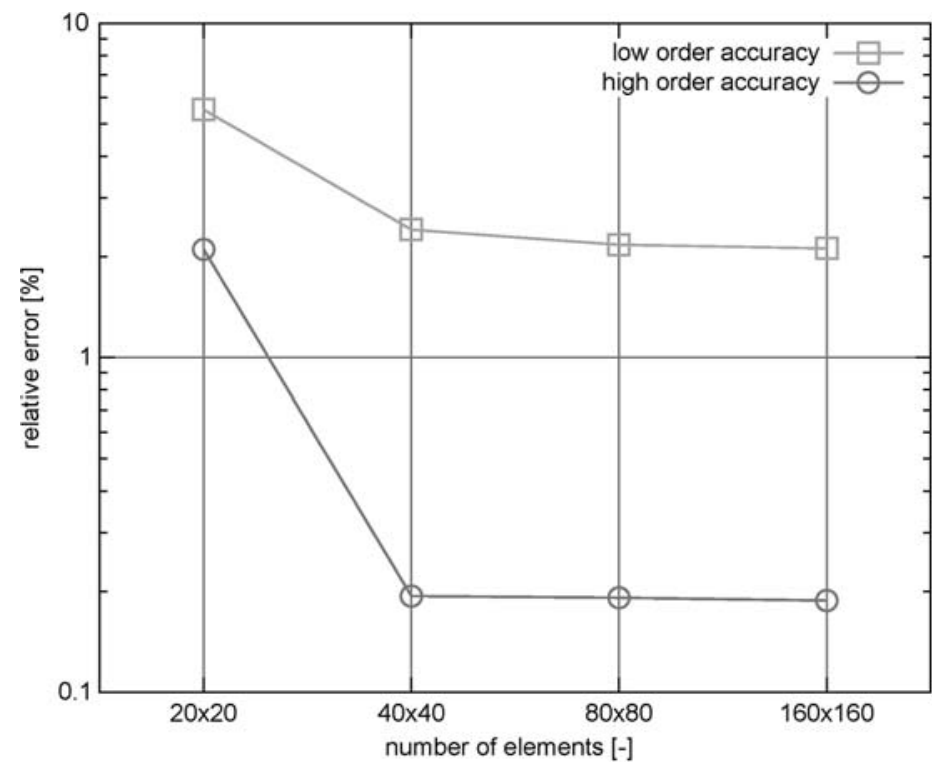

Figure 1. Convergence for finite differences with a varying $\kappa$. 


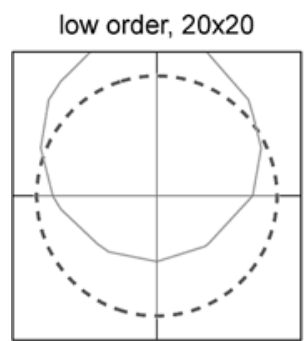

high order, $20 \times 20$

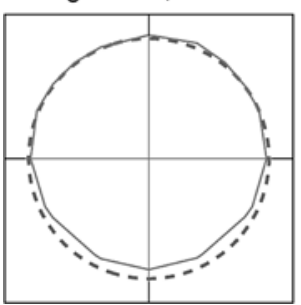

low order, $40 \times 40$

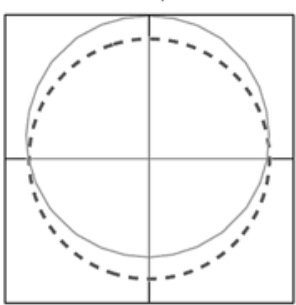

high order, $40 \times 40$

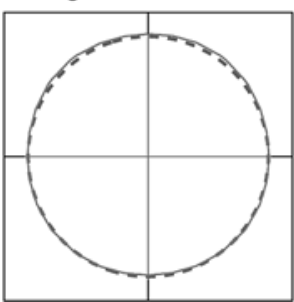

low order, $80 \times 80$

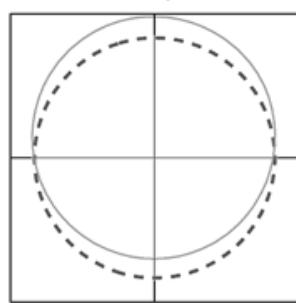

high order, $80 \times 80$

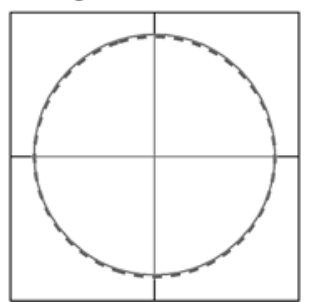

low order, $160 \times 160$

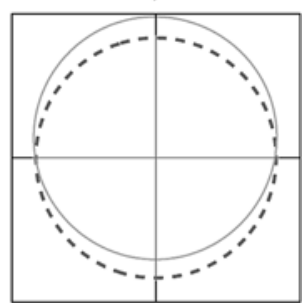

high order, $160 \times 160$

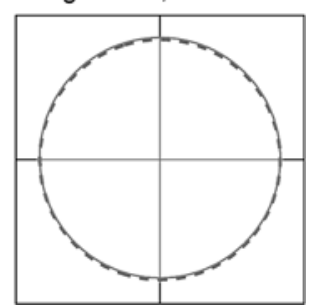

Figure 2. Comparison of numerical solution and theoretic solution for finite differences with a varying $\kappa$.

presented in Figure 4. In this case, there is no lower limit to the relative error. The high-order scheme presents a higher convergence rate than the lower order.

For the high-order scheme, a good solution, with an error around .1\%, can be obtained with a coarse mesh of 40 by 40 elements ( 41 by 41 nodes), which correspond to a discretisation of the circle over about 10 nodes. In the low-order case, the grid should be finer to obtain high-quality solution. Although difficult to compare, the obtained results appear to be much better than the results obtained by Sethian (1999) for the same type of test cases.

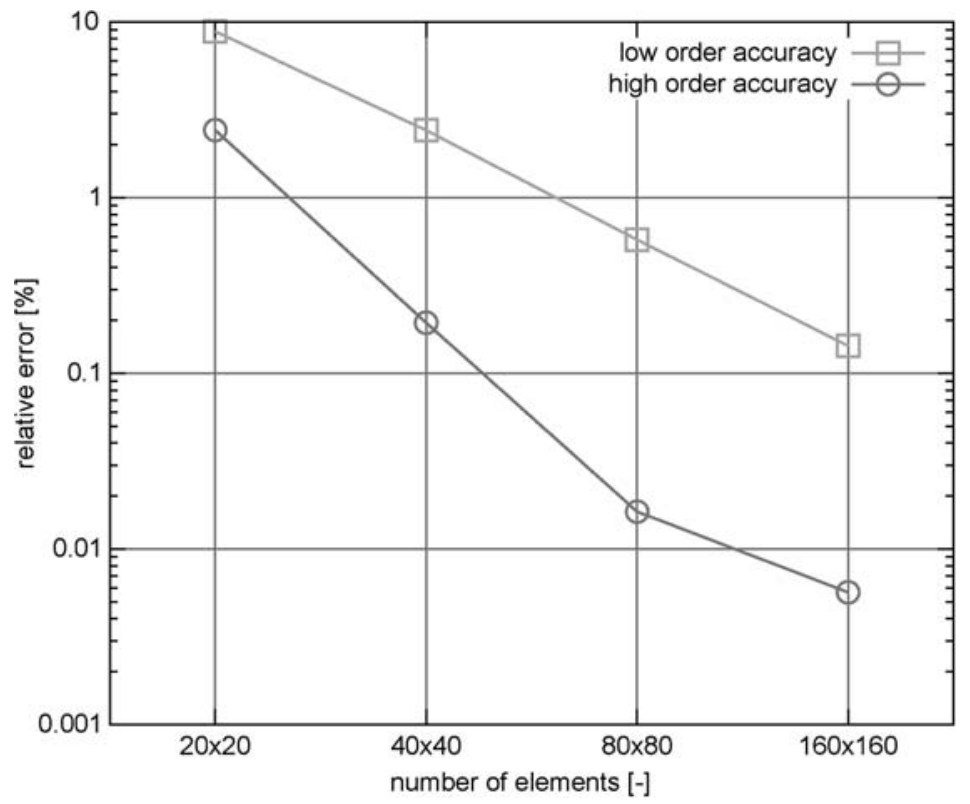

Figure 3. Convergence for finite differences with a constant $\kappa$. 


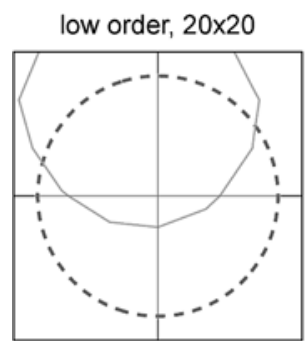

high order, $20 \times 20$

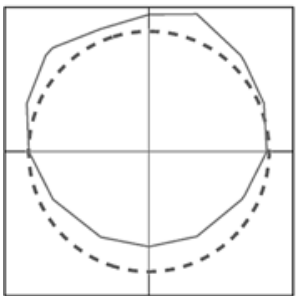

low order, $40 \times 40$

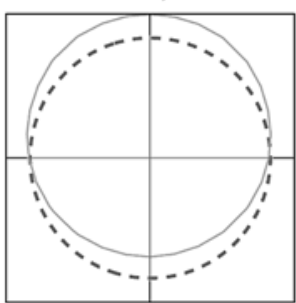

high order, $40 \times 40$

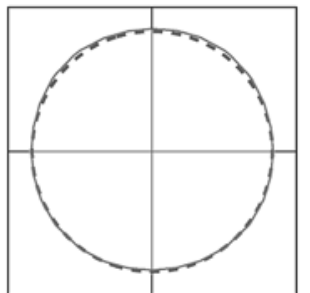

low order, $80 \times 80$

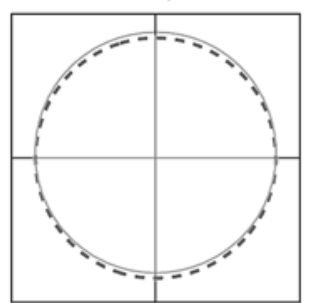

high order, $80 \times 80$

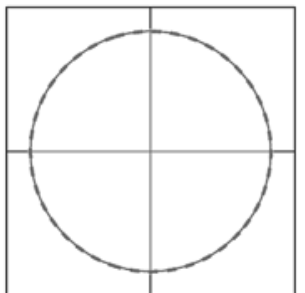

low order, $160 \times 160$

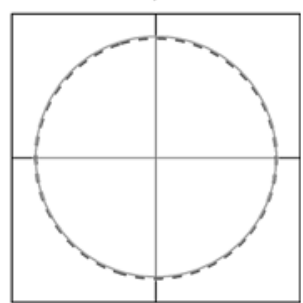

high order, $160 \times 160$

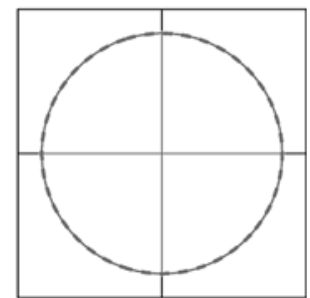

Figure 4. Comparison of numerical solution and theoretic solution for finite differences with a constant $\kappa$.

\subsection{Iso-geometry results}

Owing to the study done in finite differences, we have only looked at the case of constant interface thickness in the case of the iso-geometric formulation. This formulation has been tested with closed regularly spaced knots vector. Two orders of continuity have been studied, namely a $\mathcal{C}^{2}$ and a $\mathcal{C}^{3}$ projection space.

The evolution of the error with respect to the continuity, as depicted in Figure 5, suggests that the convergence is the same in both cases. Within this background, a better solution can

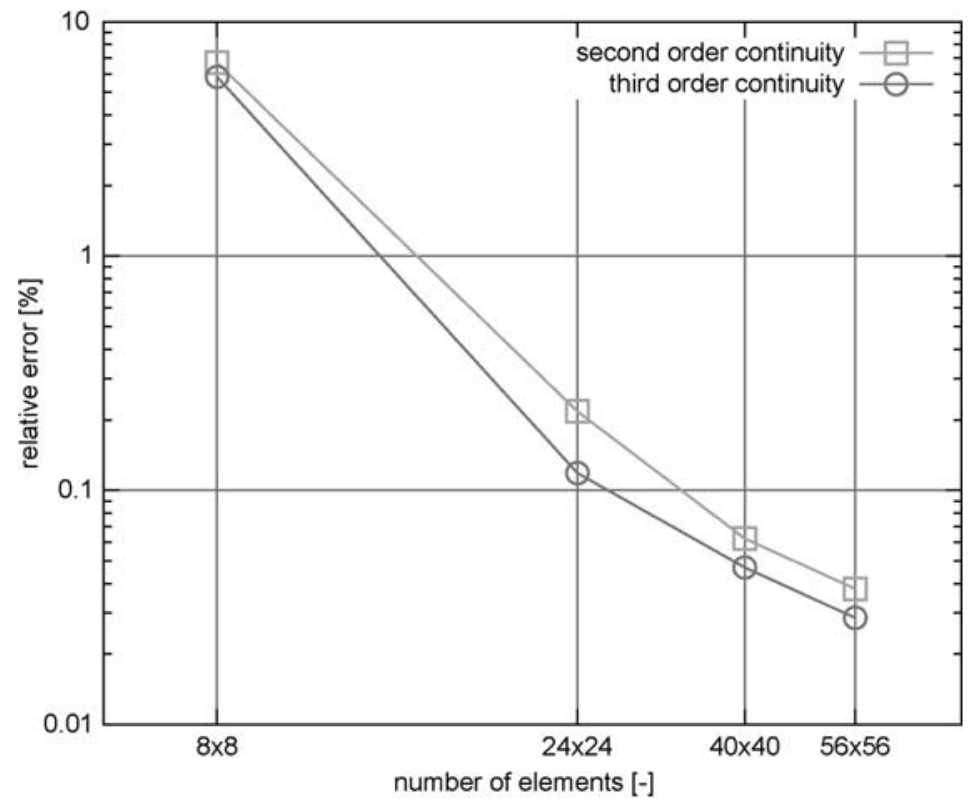

Figure 5. Convergence for iso-geometry with a constant $\kappa$. 

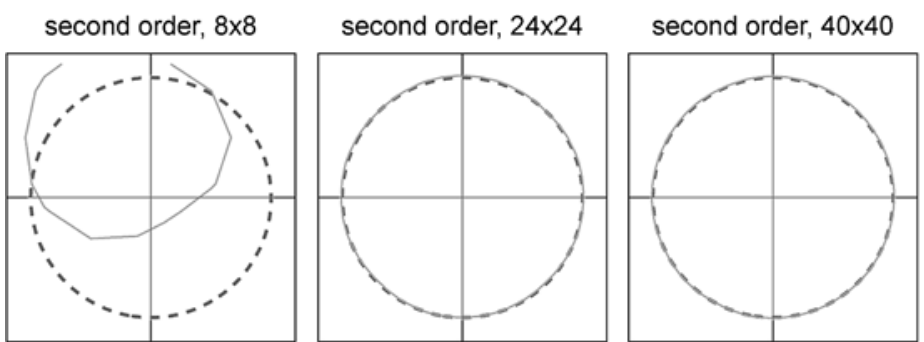

third order, $8 \times 8$

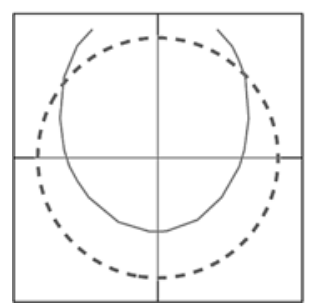

third order, $24 \times 24$

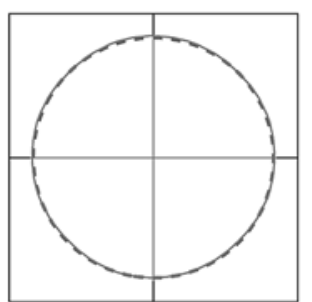

third order, $40 \times 40$

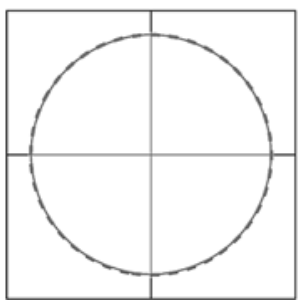

second order, $56 \times 56$

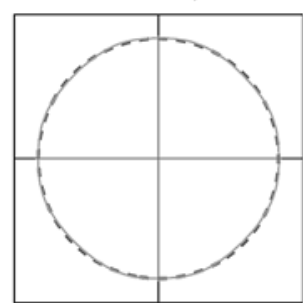

third order, $56 \times 56$

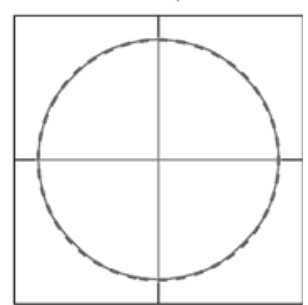

Figure 6. Comparison of numerical solution and theoretic solution for iso-geometry with a constant $\kappa$.

be achieved with a coarser discretisation: already with second-order continuity and only 24 by 24 elements, the solution is as good as the one obtained in finite differences at high order with 80 by 80 elements, although the error is measured with a different norm. The quality of the solution can also be checked in Figure 6.

\section{Conclusion}

We have presented in this paper a numerical non-local approach able to track and propagate a contour with a given velocity. The proposed scheme is a breakthrough with precedent approaches in the sense that it simplifies drastically the algorithmic complexity to solve such a problem by suppressing correction steps.

With the proposed scheme, the solution is, by construction, ensured to remain within a space that corresponds to the definition of an implicit surface. Finally, we have demonstrated that this approach can be implemented either in a finite difference approach or in a Galerkin formulation.

The actual choice of the interface thickness is to remain a user question and is a trade-off between the size of the object to be modelled and the quality of the problem solution. Finally, although it has not been tested, there is no doubt that approaches reducing the computational cost such that reducing computations on a finite band around the interface can also be applied to this approach.

\section{Acknowledgement}

The authors would like to thank the Swiss Nuclear society for its financial support.

\section{References}

Bazilevs, Y., Calo, V.M., Zhang, Y., \& Hughes, T.J.R. (2006). Isogeometric fluid-structure interaction analysis with applications to arterial blood flow. Computational Mechanics, 38(4), 310-322.

Caginalp, G. (1986). An analysis of a phase field model of a free boundary. Archive for Rational Mechanics and Analysis, 92(3), 205-245. 
Cahn, J., \& Allen, S. (1977). A microscopic theory for domain wall motion and its experimental verification in Fe-AI alloy domain growth kinetics. Journal de Physique (Colloq. 7), 38, 51-55.

Cash, J. R., \& Karp, A. H. (1990). A variable order Runge-Kutta method for initial value problems with rapidly varying right-hand sides. ACM Transactions on Mathematical Software (TOMS), 16(3), 201-222.

Chessa, J., Smolinski, P., \& Belytschko, T. (2002). The extended finite element method (XFEM) for solidification problems. International Journal for Numerical Methods in Engineering, 53(8), 1959-1977.

Chung, J., \& Hulbert, G. M. (1993). Time integration algorithm for structural dynamics with improved numerical dissipation: The generalized-alpha method. Journal of Applied Mechanics, Transactions ASME, 60(2), 371-375.

Coret, M., Valance, S., Laniel, R., \& Réthoré, J. (2009). Étude mécanique d'un changement de phase allotropique à l'échelle mésoscopique [Mechanical study of allotropic phase change at mesoscale]. Matériaux \& Techniques, 97, 81-87.

Goldman, R. (2005). Curvature formulas for implicit curves and surfaces. Computer Aided Geometric Design, 22(7), 632-658.

Gómez, H., Calo, V., Bazilevs, Y., \& Hughes, T. (2008). Isogeometric analysis of the Cahn-Hilliard phasefield model. Computer Methods in Applied Mechanics and Engineering, 197(49-50), 4333-4352.

Gravouil, A., Moës, N., \& Belytschko, T. (2002). Non-planar 3D crack growth by the extended finite element and level sets-Part II: Level set update. International Journal for Numerical Methods in Engineering, 53(11), 2569-2586.

Gustafsson, K. (1991). Control-theoretic techniques for stepsize selection in explicit Runge-Kutta methods. ACM Transactions on Mathematical Software (TOMS), 17(4), 533-554.

Gustafsson, K. (1994). Control-theoretic techniques for stepsize selection in implicit Runge-Kutta methods. ACM Transactions on Mathematical Software (TOMS), 20(4), 496-517.

Harari, I., \& Dolbow, J. (2010). Analysis of an efficient finite element method for embedded interface problems. Computational Mechanics, 46(1), 205-211.

Jansen, K. E., Whiting, C. H., \& Hulbert, G. M. (2000). A generalized- $\alpha$ method for integrating the filtered Navier-Stokes equations with a stabilized finite element method. Computer Methods in Applied Mechanics and Engineering, 190(3-4), 305-319.

Osher, S., \& Sethian, J. (1988). Fronts propagating with curvature-dependent speed: Algorithms based on Hamilton-Jacobi formulations. Journal of Computational Physics, 79, 12-49.

Sethian, J. (1999). Level set methods and fast marching methods (2nd ed.). Cambride: Cambridge University Press.

Söderlind, G. (2006). Time-step selection algorithms: Adaptivity, control, and signal processing. Applied Numerical Mathematics, 56(3), 488-502.

Valance, S., de Borst, R., Réthoré, J., \& Coret, M. (2008). A partition-of-unity-based finite element method for level sets. International Journal for Numerical Methods in Engineering, 76(10), 1513-1527. 